\title{
Traveling salesman path problems
}

\author{
Fumei Lam • Alantha Newman
}

Received: 7 November 2005 / Accepted: 15 August 2006 / Published online: 1 November 2006 (C) Springer-Verlag 2006

\begin{abstract}
In the traveling salesman path problem, we are given a set of cities, traveling costs between city pairs and fixed source and destination cities. The objective is to find a minimum cost path from the source to destination visiting all cities exactly once. In this paper, we study polyhedral and combinatorial properties of a variant we call the traveling salesman walk problem, in which the objective is to find a minimum cost walk from the source to destination visiting all cities at least once. We first characterize traveling salesman walk perfect graphs, graphs for which the convex hull of incidence vectors of traveling salesman walks can be described by linear inequalities. We show these graphs have a description by way of forbidden minors and also characterize them constructively. We also address the asymmetric traveling salesman path problem (ATSPP) and give a factor $O(\sqrt{n})$-approximation algorithm for this problem.
\end{abstract}

Mathematics Subject Classification (2000) $\quad 68 \mathrm{Q} 25 \cdot 68 \mathrm{R} 10 \cdot 90 \mathrm{C} 05 \cdot 90 \mathrm{C} 27$

\section{Introduction}

The traveling salesman problem (TSP) is a well-studied problem in combinatorial optimization. The books $[10,15]$ provide a compendium of results and

Alantha Newman was supported in part by NSF grant CCR0307536.

F. Lam $(\varangle)$

Department of Mathematics, MIT, Cambridge, MA, USA

e-mail: lam@theory.csail.mit.edu

A. Newman

Max Planck Institut für Informatik, Saarbrucken, Germany

e-mail: alantha.newman@gmail.com 
history on the problem. In this paper, we study a generalization of the problem which has not received much attention, the traveling salesman path problem (TSPP). Given an undirected graph $G=(V, E)$, a cost function on the edges, and two nodes $s, t \in V$, the TSPP is to find a Hamiltonian path from $s$ to $t$ visiting all cities exactly once. Note that nodes $s$ and $t$ need not be distinct; however the case $s=t$ is equivalent to the TSP.

A common approach for studying the TSP is to use polyhedral methods. For each traveling salesman path $P$, we associate a vector $x^{P} \in \mathbb{R}^{E}$, where edge variable $x_{e}^{P}$ takes value 1 if $e$ appears in path $P$ and 0 otherwise. For any set $S \subset V$, let $\delta(S)$ denote the set of edges with exactly one endpoint in $S$ and for a set $F \subseteq E$, let $x(F)=\sum_{e \in F} x_{e}$. Then the problem of finding a min cost $s-t$ traveling salesman path can be captured by the following integer program:

$$
\begin{array}{rcl}
\min & \sum_{e \in E} c_{e} x_{e} & \\
\text { subject to } & x(\delta(S)) \geq 1 & \text { if }|\{s, t\} \cap S|= \\
& x(\delta(S)) \geq 2 & \text { if }|\{s, t\} \cap S|= \\
x(\delta(v))=2 & \text { for all } v \in V \backslash \\
x(\delta(s))=x(\delta(t))=1 & \text { if } s \neq t \\
x(\delta(s))=x(\delta(t))=2 & \text { if } s=t \\
x_{e} \in\{0,1\} & \text { for all } e \in E .
\end{array}
$$

We obtain a linear program by replacing the integrality constraints (7) with constraints $0 \leq x_{e} \leq 1$.

One problem that arises from restricting the traveling salesman route to Hamiltonian paths is that the shortest way to visit all the vertices of $G$ may not be a simple path, i.e., may visit some vertices or edges multiple times. Another problem, arising from the linear programming relaxation, is that the polytope defined by the constraints of the linear program is not full dimensional. We resolve these problems by relaxing the condition of visiting every vertex exactly once and define an $s-t$ traveling salesman walk (TSW) (or $s-t$ walk for short) as a walk from $s$ to $t$ visiting all vertices at least once possibly with multiple visits to edges or vertices. The TSW problem asks for the minimum cost $s-t$ traveling salesman walk. This is equivalent to the traveling salesman path problem on the metric completion of $G$, where the cost between any pair of cities is the cost of the shortest path connecting the cities. In the case $s=t$, we will call an $s-t$ walk a graphical traveling salesman tour (following the terminology of [6]).

\subsection{Notation and definitions}

Let $X(G, s, t)$ denote the set of $s-t$ traveling salesman walks. For each walk $W \in X(G, s, t)$, we associate a vector $x^{W} \in \mathbb{R}^{E}$ such that $x_{e}^{W}$ represents the number of times edge $e$ appears in $W$. We will identify a walk and the vector which represents it. The $s-t$ traveling salesman walk polyhedron is the convex hull of all vectors $x^{W}$ with $W$ ranging over all $s-t$ traveling salesman walks of 
$G$. If $s=t$, the polyhedron $\operatorname{conv}(X(G, s, t))$ is the graphical traveling salesman tour polyhedron studied in [6].

Note that if $x^{W}$ is an $s-t$ traveling salesman walk of $G$, then so is $x^{W}+2 \chi_{e}$ for any edge $e$ (where $\chi_{e}$ denotes the characteristic vector for edge $e$ ). Therefore, the traveling salesman walk polyhedron of $G$ is an unbounded polyhedron if $G$ is connected.

The fractional traveling salesman walk polyhedron for a graph $G$ with fixed vertices $s$ and $t$ is defined by

$P(G, s, t)=\left\{\begin{array}{cll}x(\delta(S)) \geq 1 & \text { if }|\{s, t\} \cap S|=1 & \text { for } S \subsetneq V, S \neq \emptyset \\ x \in \mathbb{R}^{E}: x(\delta(S) \geq 2 & \text { if }|\{s, t\} \cap S|=0 \text { or } 2 & \text { for } S \subsetneq V, S \neq \emptyset \\ x \geq 0 & \end{array}\right\}$.

Note that not all integral points in $P(G, s, t)$ correspond to $s-t$ traveling salesman walks, as shown by the following example.

Example 1.1 Consider the 6-cycle $C_{6}$ with $s$ and $t$ at distance 3. The assignment $x_{e}^{*}=1$ for all edges $e$ is an integral solution in $P(G, s, t)$ but does not correspond to an $s-t$ traveling salesman walk.

\subsection{Our results}

In this paper, we address two aspects of the traveling salesman walk problem. The first is inspired by the work of Fonlupt and Naddef which characterizes the set of graphs for which the extreme points of the fractional graphical traveling salesman polyhedron are graphical traveling salesman tours [6]. These graphs are called TSP-perfect and are characterized by a list of forbidden minors. For such graphs, the graphical TSP polyhedron and fractional graphical TSP polyhedron have the same extreme points, implying that the graphical TSP polyhedron has a known description by linear inequalities and therefore, the graphical TSP can be solved in polynomial time.

We consider the analogous problem for the TSW problem and give a complete characterization of graphs for which the extreme points of the traveling salesman walk polyhedron correspond to traveling salesman walks. Our characterization of these walk-perfect graphs is also by forbidden minors. In Sect. 3, we give a constructive description for this set of graphs and in Sect. 4, we use this description to prove our main theorem. In Sect. 5, we give an alternate proof of the characterization of these graphs based on the characterization of TSP-perfect graphs from [6].

Next, we consider approximation algorithms for traveling salesman walk problems. For the symmetric traveling salesman walk problem, Hoogeveen [12] studies approximation algorithms for walks on metric instances. For fixed $s$ and $t$, he gives a 5/3-approximation for the minimum cost $s-t$ traveling salesman walk and for fixed $s$ (and varying endpoint), he gives a 3/2-approximation for the minimum cost traveling salesman walk starting at $s$. 
We address the asymmetric version of the traveling salesman walk problem (ATSW), in which edge costs satisfy the triangle inequality but may be asymmetric (i.e. $c_{i j} \neq c_{j i}$ ). For the case $s=t$, Frieze et al. [7] give a $\log n$-approximation algorithm. In Sect. 6, we use similar methods to give the first non-trivial ATSW approximation algorithm, with approximation factor $O(\sqrt{n})$.

\section{Walk-perfection}

In this section, we introduce the notion of walk-perfection of a graph. We first review previous work on TSP-perfect graphs. For graph $G$, a graphical traveling salesman tour, or tour for short, is a connected multigraph with even degree at every vertex. Let $X_{\mathrm{TSP}}(G)$ denote the set of graphical traveling salesman tours of $G$ and consider the fractional graphical traveling salesman polyhedron

$$
P(G)=\left\{\begin{array}{c}
x \in \mathbb{R}^{E}: x(\delta(S)) \geq 2 \quad \text { for } S \subsetneq V, S \neq \emptyset \\
x \geq 0
\end{array}\right\} .
$$

Properties of this polyhedron and other combinatorial results have been studied in [4-6,16,17]. Clearly, $\operatorname{conv}\left(X_{\mathrm{TSP}}(G)\right) \subseteq P(G)$; however, there are graphs for which the inclusion is strict. A graph $G$ is TSP-perfect if $\operatorname{conv}\left(X_{\mathrm{TSP}}(G)\right)=$ $P(G)$, i.e., the vertices of the polyhedron are graphical traveling salesman tours. Note that the equality always holds for disconnected graphs $G$, since in this case, both the convex hull of tours and the fractional graphical TSP polyhedron are the empty set. Therefore, all disconnected graphs are TSP-perfect.

A minor of a graph $G=(V, E)$ is a graph that can be obtained from $G$ by a sequence of edge deletions (denoted $G \backslash\{e\}$ ) and edge contractions (denoted G.e). A graph $G$ is $H$ minor free if $G$ does not contain $H$ as a minor. Fonlupt and Naddef show that there is a forbidden minor characterization of TSP-perfect graphs using the graphs $M_{1}, M_{2}, M_{3}$ in Fig. 1.

Theorem 2.1 [6] A connected graph $G$ is TSP-perfect if and only if $G$ is $\left[M_{1}, M_{2}\right.$, $\left.M_{3}\right]$ minor free.

We consider the analogous problem for the traveling salesman walk problem. Let $X(G, s, t)$ denote the set of $s-t$ traveling salesman walks and consider the fractional traveling salesman walk polyhedron

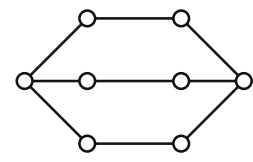

$M_{1}$

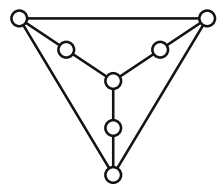

$M_{2}$

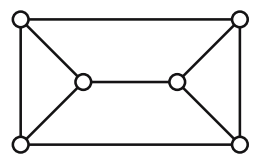

$M_{3}$

Fig. 1 Excluded minors for TSP-perfect graphs 
$P(G, s, t)=\left\{\begin{array}{cll}x(\delta(S)) \geq 1 & \text { if }|\{s, t\} \cap S|=1 & \text { for } S \subsetneq V, S \neq \emptyset \\ x \in \mathbb{R}^{E}: x(\delta(S)) \geq 2 & \text { if }|\{s, t\} \cap S|=0 \text { or } 2 & \text { for } S \subsetneq V, S \neq \emptyset \\ x \geq 0 & & \end{array}\right\}$.

As with the traveling salesman problem, there are graphs for which the inclusion $\operatorname{conv}(X(G, s, t)) \subseteq P(G, s, t)$ is strict. Our goal is to characterize graphs $G$ for which equality holds for any choice of $s$ and $t$.

Definition 2.2 A graph $G$ is $s-t$ walk-perfect if $P(G, s, t)=\operatorname{conv}(X(G, s, t))$ and $G$ is walk-perfect if it is $s-t$ walk-perfect for all choices of $s$ and $t$.

As in the case of TSP-perfection, any disconnected graph $G$ satisfies $\operatorname{conv}((X(G, s, t))=P(G, s, t)$. Therefore, all disconnected graphs are walk-perfect and we focus our attention on characterizing the set of connected walkperfect graphs.

In Example 1.1, if all edge costs in the 6-cycle are equal to a fixed positive value, then $x^{*}$ is an optimal solution over $P(G, s, t)$ that does not correspond to an $s-t$ traveling salesman walk. This shows that $C_{6}$ with $s$ and $t$ at distance 3 is not $s-t$ walk-perfect and therefore, $C_{6}$ is not walk-perfect. A graph is called minimally non walk-perfect if it is not walk-perfect but all of its proper minors are walk-perfect. Our main theorem, which we prove in the next two sections, states that $C_{6}$ is the only minimally non walk-perfect graph.

Theorem 2.3 (Main Theorem) A connected graph $G$ is walk-perfect if and only if $G$ is $C_{6}$ minor free.

In [6], Fonlupt and Naddef prove their main result by characterizing properties of minimally non TSP-perfect graphs, graphs which are not TSP-perfect but all of whose minors are TSP-perfect. They show the only minimally non TSPperfect graphs are the graphs $M_{1}, M_{2}, M_{3}$ in Fig. 1 and leave as an open problem a direct characterization of TSP-perfection using the structure of $\left[M_{1}, M_{2}, M_{3}\right]$ minor free graphs. One of the goals of our research was to address this open problem. As an initial step, we need a proof characterizing walk-perfect graphs which is independent of the results of Fonlupt and Naddef. We therefore include two proofs of our main result. The first proof leads to an independent constructive characterization of TSP-perfect graphs, thus settling the aforementioned open question of Fonlupt and Naddef [14]. The second proof relies directly on the results of Fonlupt and Naddef, but is more concise.

\section{$3 C_{6}$ Minor free graphs}

In this section, we give a constructive characterization of the set of $C_{6}$ minor free graphs. We will use this characterization in the first proof of our main theorem. 


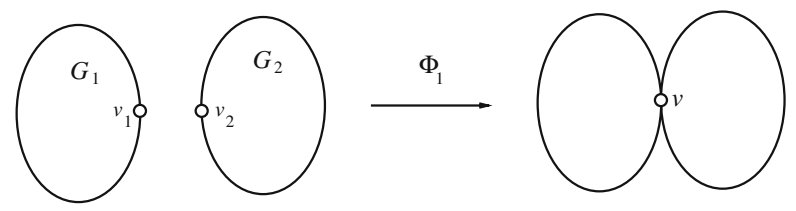

Fig. 2 Operation $\Phi_{1}$

We first show that we can reduce our problem to the characterization of 2-connected walk-perfect graphs. Suppose $G_{1}$ and $G_{2}$ are connected graphs with specified vertices $s_{1}, t_{1} \in V\left(G_{1}\right)$ and $s_{2}, t_{2} \in V\left(G_{2}\right)$. Let $v_{1} \in V\left(G_{1}\right)$ and $v_{2} \in V\left(G_{2}\right)$ be chosen so that at least two of $s_{1}, s_{2}, t_{1}, t_{2}$ are equal to $v_{1}$ or $v_{2}$. The operation $\Phi_{1}$ identifies vertices $v_{1}$ and $v_{2}$ to obtain graph $G$ (see Fig. 2) with cut vertex $v$. If the set $\left\{s_{1}, s_{2}, t_{1}, t_{2}\right\} \backslash\left\{v_{1}, v_{2}\right\}$ has two vertices, then relabel these vertices by $s$ and $t$. If it has one vertex, then relabel this vertex by $s$ and let $t=v$; if it has no vertices, then let $s=v$ and $t=v$.

Every 1-connected graph can be built by repeated applications of operation $\Phi_{1}$ from blocks which are either 2-connected graphs or single edges. In Lemma 4.6, we will show that walk-perfection of a graph is preserved under operation $\Phi_{1}$ and therefore, we can focus our attention on the characterization of 2-connected walk-perfect graphs.

An ear decomposition $G_{1}, G_{2}, \ldots G_{m}=G$ of a graph $G$ is a sequence of subgraphs starting from a simple graph $G_{1}$ (a vertex, edge or cycle) such that for each $i, G_{i+1}$ is obtained from $G_{i}$ by adding an ear. The operation of adding an ear is performed by choosing two vertices $u$ and $v$ (the endpoints of the ear) from $G_{i}$ and adding a path from $u$ to $v$ using new vertices (or no vertices if the path is edge $(u, v))$. If $u \neq v$, the ear is proper and a proper ear decomposition is one in which every ear operation is proper. The following theorem is due to Robbins [18].

Theorem 3.1 $G$ is 2-connected if and only if $G$ has a proper ear decomposition starting from any cycle of $G$.

One particular ear operation is duplication of a degree-2 vertex. In such an operation, for a vertex $u$ of degree 2 in $G_{i}$ with neighborhood $N(u)=\{a, b\}$, duplication of $u$ results in a graph $G_{i+1}$ on vertices and edges

$$
\begin{aligned}
& V\left(G_{i+1}\right)=V\left(G_{i}\right) \cup\left\{u^{\prime}\right\} \\
& \left.E\left(G_{i+1}\right)=E\left(G_{i}\right) \cup\left\{\left(a, u^{\prime}\right),\left(u^{\prime}, b\right)\right\}\right) .
\end{aligned}
$$

Fig. 3 Vertex duplication of a degree- 2 vertex
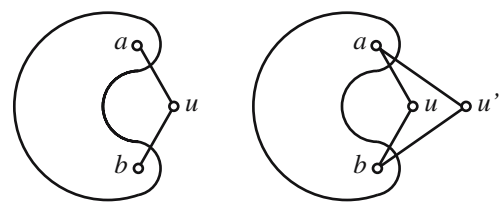
Fig. 4 Examples of graphs in $\mathcal{T}$
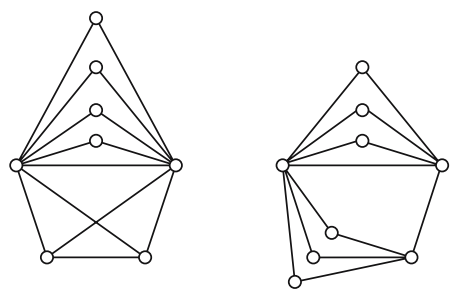

Let $K_{5}$ denote the complete graph on five vertices and consider the class $\mathcal{T}$ of 2-connected graphs obtained from $K_{5}$ by repeated applications of the operations edge deletion, edge contraction, and duplication of degree- 2 vertices. We show that this set of graphs is exactly the set of 2-connected graphs not containing a $C_{6}$ minor.

Theorem 3.2 A 2-connected graph $G$ is $C_{6}$ minor free if and only if $G \in \mathcal{T}$.

Proof Since $K_{5}$ does not contain a 6-cycle and the size of the largest cycle cannot increase under edge deletion, contraction, or vertex duplication, no graph in $\mathcal{T}$ contains a $C_{6}$ minor.

Conversely, suppose $G$ is 2-connected and $C_{6}$ minor free. We will show $G \in \mathcal{T}$ by showing that there is an ear decomposition of $G$ starting with a minor of $K_{5}$ such that each ear operation corresponds to edge addition or vertex duplication of a degree-2 vertex. By Theorem 3.1, $G$ has a proper ear decomposition $G_{1}, G_{2}, \ldots G_{m}=G$ and we can choose the initial graph $G_{1}$ in the decomposition to be the largest cycle $C_{k}=\left\{v_{1}, v_{2}, \ldots v_{k}\right\}$ in $G$ ( $k \leq 5$ by assumption). The edges $\left(v_{i}, v_{i+1}\right)$ for $i=1,2, \ldots k-1$ and $\left(v_{k}, v_{1}\right)$ will be called cycle edges and the edges $\left(v_{i}, v_{j}\right)$ with $j \neq i-1, i+1(\bmod k)$ will be called chords. If there are $j-1$ induced chords in $G$ between vertices $v_{1}, v_{2}, \ldots v_{k}$, let $G_{j}$ denote the cycle $v_{1}, v_{2}, \ldots v_{k}$ together with all induced chords and let $a, b \in\left\{v_{1}, \ldots v_{k}\right\}$ be the two vertices that are endpoints for the next ear operation. Because we have already included all chords, the next ear cannot be edge $(a, b)$. Also, note that the length of the longest path between $a$ and $b$ in $G_{j}$ is at least $\left\lceil\frac{k}{2}\right\rceil$, so if the next ear is a path of length at least 3 , then it would create a cycle of length at least $\left\lceil\frac{k}{2}\right\rceil+3>k($ since $k \leq 5)$, a contradiction to our choice of $k$. Therefore, it must be a path of length 2 which consists of an additional vertex $u^{\prime}$ and edges $\left(a, u^{\prime}\right),\left(u^{\prime}, b\right)$. Now, if $(a, b)$ is a cycle edge in $C_{k}$, then the longest path from $a$ to $b$ has length $k-1$, so adding an ear of length 2 would create a $k+1$ cycle, a contradiction. Therefore, $(a, b)$ cannot be a cycle edge (but $a$ and $b$ may be connected by a chord). Since $k \leq 5, a$ and $b$ have a common neighbor, say $u$.

Claim: $\operatorname{deg}_{G_{j}}(u)=2$, i.e., the neighborhood of $u$ in $G_{j}$ is $N_{G_{j}}(u)=\{a, b\}$. Otherwise, let $w \in N_{G_{j}}(u) \backslash\{a, b\}$. Since $k \leq 5, w$ must also be adjacent to either $a$ or $b$, say $a$. Then the cycle formed by concatenating the path $(w, u),(u, a),\left(a, u^{\prime}\right)$, $\left(u^{\prime}, b\right)$ and the path from $b$ to $w$ (along $G_{1}$, but not through $a$ ) has length at least $k+1$, which is a contradiction (see Fig. 5).

Therefore, $u$ has degree 2 in $G_{j}$ and the operation of adding vertex $u^{\prime}$ and edges $\left(a, u^{\prime}\right),\left(u^{\prime}, b\right)$ corresponds to vertex duplication of $u$. Note that since $\left(G_{j} \backslash\{u\}\right) \cup\left\{u^{\prime}\right\}=G_{j}$, the same argument shows we cannot add a path $p$ of 
Fig. 5 Forbidden adjacencies in the ear operation
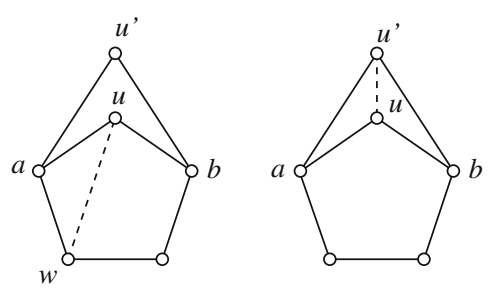

any length from either $u$ or $u^{\prime}$ to any other vertex in $G_{j} \backslash\{a, b\}$. Similarly, we cannot add a path $p$ of any length between $u$ and $u^{\prime}$ (denoted $u \stackrel{p}{\rightarrow} u^{\prime}$ ), since the cycle formed by concatenating the path $(a, u), u \stackrel{p}{\rightarrow} u^{\prime},\left(u^{\prime}, b\right)$ and the path of $k-2$ cycle edges from $b$ to $a$ has length at least $k+1$ (see Fig. 5). Therefore, neither $u$ nor $u^{\prime}$ can be chosen as endpoints of the next ear. This implies we must always use vertices among $\left\{v_{1}, v_{2}, \ldots v_{k}\right\}$ as ear endpoints and each ear operation corresponds to duplicating a vertex. Since $G_{1}$ is a minor of $K_{5}$, it follows that $G \in \mathcal{T}$.

This theorem gives us a constructive characterization of the set of 2connected $C_{6}$ minor free graphs. Note that the proof of Theorem 3.2 also shows the following.

Corollary 3.3 Suppose $G \in \mathcal{T}$ is obtained from $K_{5}$ by a sequence of edge deletions, contractions and degree-2 vertex duplications. Then first performing all edge deletions and contractions followed by any permutation of the degree- 2 vertex duplications also results in graph $G$.

From this corollary, if graph $G \in \mathcal{T}$ has two specified vertices $s$ and $t$ which result from the duplication of a degree- 2 vertex $u$, then we can reorder the vertex duplications so that the duplication of $u$ to obtain $s$ and $t$ comes first in the ordering and all other vertex duplications follow. Otherwise, if $s$ and $t$ do not result from the duplication of a degree- 2 vertex, we can assume that $s$ and $t$ are vertices in the initial subgraph of $K_{5}$ to which the operations of edge deletion, edge contraction, and degree-2 vertex duplication are performed to obtain $G$.

\section{Characterization of walk-perfect graphs}

In this section, we will show that $C_{6}$ is the only forbidden minor in the set of 2connected traveling salesman walk-perfect graphs. Since graph $G$ has specified vertices $s$ and $t$, we first define the notion of a labeled minor of a graph. The operation of edge deletion remains the same as for unlabeled graphs. For the operation of edge contraction, if an edge $e$ is chosen for edge contraction, the resulting vertex from the contraction receives the labels of both endpoints of $e$, with possibly both labels $s$ and $t$. In the case $s$ and $t$ label the same vertex in the resulting graph, an $s-t$ traveling salesman walk is a graphical traveling salesman tour.

We first show that walk-perfection is preserved under the labeled minor operations; the proof is modeled on Fonlupt and Naddef's [6] proof that TSPperfection is preserved under the minor operations. 
Lemma 4.1 Any connected labeled minor of a connected walk-perfect graph is walk-perfect.

Proof Suppose a connected graph $G$ has specified vertices $s, t \in V(G)$ and suppose $G$ is $s-t$ walk-perfect. We show that if deletion of an edge $e$ results in a connected graph, then the minor $G \backslash\{e\}$ is $s-t$ walk-perfect. Since $G \backslash\{e\}$ is connected, $P(G \backslash\{e\}, s, t)$ is nonempty. Then let $y$ be an extreme point of $P(G \backslash\{e\}, s, t)$ and let

$$
x_{f}=\left\{\begin{aligned}
y_{f} & \text { if } f \in E \backslash\{e\}, \\
0 & \text { if } f=e .
\end{aligned}\right.
$$

Since $y$ is an extreme point of $P(G \backslash\{e\}, s, t)$ and since $x$ has one more variable and one more linearly independent tight constraint than $y, x$ is an extreme point in $P(G, s, t)$. By $s-t$ walk-perfection of $G, x$ is an $s-t$ traveling salesman walk in $G$, and since $x$ does not use edge $e, y$ is an $s-t$ traveling salesman walk in $G \backslash\{e\}$. Thus, $G \backslash\{e\}$ is $s-t$ walk-perfect.

Now, for the edge contraction operation, if $G$ is connected, then G.e is connected, so for any vertices $s$ and $t, P(G . e, s, t)$ is nonempty. Let $y$ be an extreme point of $P(G . e, s, t)$ and let

$$
\bar{x}_{f}= \begin{cases}y_{f} & \text { if } f \in E \backslash\{e\}, \\ 0 & \text { if } f=e .\end{cases}
$$

Consider cuts $\delta\left(W^{\prime}\right)$ of $G$ containing $e$ such that $s$ and $t$ are on the same side of the cut and let $\alpha=\min \bar{x}\left(\delta\left(W^{\prime}\right)\right)$. Similarly, consider cuts $\delta\left(W^{\prime \prime}\right)$ of $G$ containing $e$ such that $s$ and $t$ fall on different sides of the cut and let $\beta=\min \bar{x}\left(\delta\left(W^{\prime \prime}\right)\right)$. Now, let

$$
x_{f}= \begin{cases}y_{f} & \text { if } f \in E \backslash\{e\}, \\ \max \{0,2-\alpha, 1-\beta\} & \text { if } f=e .\end{cases}
$$

Note that $x \in P(G, s, t)$ since any cut $\delta\left(W^{\prime}\right)$ containing $e$ that does not separate $s$ and $t$ satisfies $x\left(\delta\left(W^{\prime}\right)\right) \geq 2$, any cut $\delta\left(W^{\prime \prime}\right)$ containing $e$ that separates $s$ and $t$ satisfies $x\left(\delta\left(W^{\prime \prime}\right)\right) \geq 1$, and any cut not containing $e$ is also a cut in G.e.

Let $\theta(x)$ and $\theta(y)$ denote the set of tight constraints for $x$ and $y$. By possibly taking complements, we can assume any tight constraint $C$ in $\theta(y)$ does not contain the vertex resulting from contraction of edge $e$. Then $C$ is also a tight constraint for $x$. Since any tight edge constraint for $y$ is also tight for $x$, it follows that $x$ is defined by $\theta(y)$ and

$$
\begin{aligned}
x_{e}=0 & \text { if } \alpha \geq 2 \text { and } \beta \geq 1 \\
x\left(\delta\left(\bar{W}^{\prime}\right)\right)=2 & \text { if } \alpha<2 \text { and } 2-\alpha \geq 1-\beta \\
x\left(\delta\left(\bar{W}^{\prime \prime}\right)\right)=1 & \text { if } \beta<1 \text { and } 1-\beta \geq 2-\alpha,
\end{aligned}
$$


where $\bar{W}^{\prime}=\arg \min \bar{x}\left(\delta\left(W^{\prime}\right)\right)$ and $\bar{W}^{\prime \prime}=\arg \min \bar{x}\left(\delta\left(W^{\prime \prime}\right)\right)$. Since $x$ has one more variable and one more linearly independent tight constraint, it is an extreme point of $P(G, s, t)$ and therefore an $s-t$ traveling salesman walk in $G$ (by $s-t$ walk-perfection of $P(G, s, t))$. Therefore, $y$ is an $s-t$ traveling salesman walk in G.e, implying $G$.f is $s-t$ walk-perfect.

We first prove walk-perfection for graph $K_{5}$ by enumerating the extreme points of the fractional walk polyhedron using the program polymake [8]. We check that for each extreme point, the degree of every vertex $v \notin\{s, t\}$ is even and the degrees of $s$ and $t$ are odd (see [14] for a complete enumeration of extreme points). Since these conditions are satisfied, all of the extreme points correspond to $s-t$ traveling salesman walks and the lemma follows.

Lemma 4.2 $K_{5}$ is walk-perfect.

We give a second proof of this result in Sect. 5 using the characterization of TSP-perfect graphs. However, it is important to note that we have verified this lemma independently of the TSP-perfect graph characterization, as we would like a proof of our main theorem which is independent of this result.

Note that if $G$ is a connected graph, then the fractional $s-t$ walk polyhedron $P(G, s, t)$ is full dimensional (otherwise, $P(G, s, t)$ is empty). The following theorem from [9] (which we restate in our context) gives a condition for showing the extreme points of polyhedron $P(G, s, t)$ are integral.

Theorem 4.3 [9] Let $G$ be a connected graph and let $P=\{x: A x \leq b\}$ be any polyhedron with $X(G, s, t) \subset P$. Then $P=\operatorname{conv}(X(G, s, t))$ if for any non-zero cost function $c$, we can show that there exists an inequality in $\{A x \leq b\}$ satisfied at equality by all optimal solutions to $\min \{c x: x \in X(G, s, t)\}$ whenever this minimum is finite.

We use this theorem to show that walk-perfection is preserved under duplication of degree-2 vertices. Let $G$ be a $C_{6}$ minor free graph, let $s, t \in V(G)$, and consider the ear decomposition of $G$ in Theorem 3.2. If $s$ and $t$ are obtained by duplicating a vertex $u$, then by Corollary 3.3, we can reorder the vertex duplications so that the operation of duplicating $u$ to obtain $s$ and $t$ comes first in the ordering and all other vertex duplications follow. In this case, the sequence of ear operations gives graphs $G_{1}, G_{2}, \ldots G_{k}=G$, where $G_{i+1}$ is obtained from $G_{i}$ by an edge addition for $i<j, G_{j+1}$ is obtained from $G_{j}$ by duplicating $u$ to obtain $s$ and $t$ and $G_{i+1}$ is obtained from $G_{i}$ by a degree-2 vertex duplication for $i>j$. Otherwise, if $s$ and $t$ are not obtained by duplicating the same vertex, we can choose the first graph $G_{1}$ to be the largest cycle containing $s$ and $t$ and no subsequent vertex duplication relabels a new vertex as $s$ or $t$. We first show that for $i>j$ and for fixed $s, t \in V\left(G_{i}\right)$, if $G_{i}$ is $s-t$ walk-perfect, then $G_{i+1}$ is also $s-t$ walk-perfect.

Lemma 4.4 For fixed $s, t \in V(G)$, suppose $G$ is $s-t$ walk-perfect and contains a vertex $u$ of degree 2 with $N(u)=\{a, b\}$ (possibly $u=s$ or $u=t$ ). Then the graph $G^{\prime}=\left(V \cup\left\{u^{\prime}\right\}, E \cup\left\{\left(a, u^{\prime}\right),\left(u^{\prime}, b\right)\right\}\right)$ is also s-t walk-perfect. 
Proof For any cost function $c$ on $G^{\prime}$, consider the set $\mathcal{P}$ of minimum cost $s-t$ traveling salesman walks in $G^{\prime}$. If $c$ has a negative component then the optimum is not finite, so we can assume that $c$ is nonnegative. We show that there is an inequality of the fractional $s-t$ walk polyhedron satisfied at equality by all $s-t$ traveling salesman walks in $\mathcal{P}$. If $c$ does not satisfy the triangle inequality, then there is an edge $(i, j)$ such that $c_{i j}>c_{i k}+c_{k j}$ and in all optimal solutions, $x_{i j} \geq 0$ is a tight inequality.

Now, let $c$ be a cost function satisfying the triangle inequality on $G^{\prime}$, let $\mathcal{I}=N(a) \cap N(b)$ denote the set of vertices in $G^{\prime}$ adjacent to both $a$ and $b$, and for any proper subset $S$, let $f(S)=1$ if $|S \cap\{s, t\}|=1, f(S)=2$ otherwise. By abuse of notation, we will use $f(u)$ to denote $f(\{u\})$.

Case $1 c_{a w}+c_{w b}>c_{a v}+c_{v b}$ for some $v, w \in \mathcal{I}$.

If the inequality $x(\delta(w)) \geq f(w)$ is not tight for all optimal solutions $x \in \mathcal{P}$, there exists an optimal traveling salesman walk $x^{*}$ such that $x^{*}(\delta(w))>f(w)$. In this case, we show one of the non-negativity constraints $x_{a w} \geq 0$ or $x_{w b} \geq 0$ is tight for all $x \in \mathcal{P}$. If $x_{a w}^{*} \geq 1, x_{w b}^{*} \geq 1$, decreasing both values by 1 and increasing $x_{a v}^{*}, x_{v b}^{*}$ by 1 results in a $s-t$ traveling salesman walk of strictly smaller cost (since degree parity is preserved at every vertex and no vertex is disconnected), a contradiction to the optimality of $x^{*}$. Therefore, it must be the case that one of $x_{a w}^{*}$ or $x_{w b}^{*}$ is zero, say $x_{a w}^{*}=0$. Then $x_{w b}^{*} \geq 3\left(\right.$ since $x^{*}(\delta(w))>f(w)$ and the degrees of $s$ and $t$ are odd). Since another traveling salesman walk is obtained by decreasing $x_{w b}^{*}$ by 2 , the optimality of $x^{*}$ implies $c_{w b}=0$. Now, $c_{a w}>c_{a v}+c_{v b}=c_{a v}+c_{v b}+c_{w b}$, so no optimal $s-t$ traveling salesman walk uses edge $(a, w)$, implying inequality $x_{a w} \geq 0$ is tight for all $x \in \mathcal{P}$.

Case $2 c_{a v}+c_{v b}=c_{a w}+c_{w b}$ for all $v, w \in \mathcal{I}$.

Case $2.1 c_{a v}$ or $c_{v b}=0$ for some $v \in \mathcal{I} \backslash\{s, t\}$.

Without loss of generality, let $c_{v b}=0$. Then any $s-t$ traveling salesman walk in $G=G^{\prime} \backslash v$ can be extended by edge $(v, b)$ (traversed twice) to an $s-t$ traveling salesman walk in $G^{\prime}$ of the same cost. Conversely, since $c_{a v}=c_{a v}+c_{v b}=c_{a w}+c_{w b}$ for all $w \in \mathcal{I}, w \neq v$, any $s-t$ traveling salesman walk $x$ in $G^{\prime}$ can be converted into an $s-t$ traveling salesman walk $y$ in $G$ of the same cost as follows. Choose some $w \in \mathcal{I} \backslash v$ and let $y_{a w}=x_{a w}+x_{a v}$ and $y_{w b}=x_{w b}+x_{a v}$. Since the parity of degrees at all vertices remain the same and the costs of solutions $x$ and $y$ are the same, the optimal $s-t$ traveling salesman walks in $G$ and the optimal $s-t$ traveling salesman walks in $G^{\prime}$ have the same cost. Now, since $G=G^{\prime} \backslash\{v\}$ is $s-t$ walk-perfect, there exists some constraint that is tight for all optimal $s-t$ traveling salesman walks in $G$. If this is a non-negativity constraint $y_{e} \geq 0$, then constraint $x_{e} \geq 0$ is also tight for all optimal $s-t$ traveling salesman walks $x$ in $G^{\prime}$. Otherwise, it is a cut constraint $C$ and we can assume without loss of generality that $b \in C$. Then constraint $C^{\prime}=C \cup\{v\}$ is tight for every $x \in \mathcal{P}$.

Case $2.2 c_{a v}, c_{v b}>0$ for all $v \in \mathcal{I} \backslash\{s, t\}$.

We claim that for $v \in \mathcal{I} \backslash\{s, t\}$, any optimal integral solution $x^{*}$ satisfies $x^{*}(\delta(v))=f(v)=2$. To prove this, assume $x^{*}(\delta(v)) \geq 3$. If $x_{a v}^{*}$ or $x_{v b}^{*} \geq 3$, 
decreasing $x^{*}$ by 2 on this edge yields another integral solution of strictly smaller cost, contradicting minimality of $x^{*}$. Since $x^{*}(\delta(v))$ is even for $v \in \mathcal{I} \backslash\{s, t\}$, we must have $x_{a v}^{*}=x_{v b}^{*}=2$. For any other vertex $w \in \mathcal{I} \backslash v$, either $x_{a w}^{*} \geq 1$ or $x_{w b}^{*} \geq 1$, say $x_{a w}^{*} \geq 1$. Then by decreasing $x_{a w}^{*}, x_{a v}^{*}, x_{v b}^{*}$ by 1 and increasing $x_{w b}^{*}$ by 1 , we obtain another $s-t$ traveling salesman walk of strictly smaller cost, again a contradiction. Therefore, $x^{*}(\delta(v))=f(v)=2$ for all $v \in \mathcal{I} \backslash\{s, t\}$.

We have shown that performing vertex duplication on $G$ to obtain a new vertex not labelled $s$ or $t$ preserves $s-t$ walk-perfection of $G$. Now, we show walkperfection is also preserved under vertex duplication when the two resulting vertices are relabeled $s$ and $t$. Consider the ear decomposition $G_{1}, G_{2}, \ldots G_{k}=G$ discussed above, where $G_{i+1}$ is obtained from $G_{i}$ by an edge addition for $i<j$ and $G_{j+1}$ is obtained from $G_{j}$ by duplicating $u$ to obtain $s$ and $t$.

Lemma 4.5 If $G_{j}$ is walk-perfect and $G_{j+1}$ is obtained from $G_{j}$ by duplicating vertex $u$ to obtain $s$ and $t$, then $G_{j+1}$ is also walk-perfect.

Proof By construction of the ear decomposition, $G_{j}$ is obtained from the cycle $G_{1}$ by edge additions and therefore, has no other vertex duplications (i.e., is a subgraph of the graph in Fig. 6). Note that this graph is a subgraph of $K_{5}$ and is therefore walk-perfect.

Case $1 c_{a s}+c_{s b}>c_{a t}+c_{t b}$ or $c_{a s}+c_{s b}<c_{a t}+c_{t b}$

The analysis of Case 1 in Lemma 4.4 gives a tight constraint for this case.

Case $2 c_{a s}+c_{s b}=c_{a t}+c_{t b}$.

Case 2.1 One of $c_{a s}, c_{s b}, c_{a t}$ or $c_{t b}$ equals 0 .

Without loss of generality, let $c_{s b}=0$. For $\widehat{s}=b$ and $\widehat{t}=t$, any $\widehat{s}-\widehat{t}$ traveling salesman walk in $G=G^{\prime} \backslash s$ can be extended by edge $(s, b)$ to an $s-t$ traveling salesman walk in $G^{\prime}$ of the same cost. Conversely, since $c_{a s}=c_{a s}+c_{s b}=c_{a w}+c_{w b}$ for all $w \neq s$, any $s-t$ traveling salesman walk $x$ in $G^{\prime}$ can be converted into an $\widehat{s}-\widehat{t}$ traveling salesman walk $y$ in $G$ of the same cost as follows. Choose $w \in \mathcal{I} \backslash s$ and let $y_{a w}=x_{a w}+x_{a s}$ and $y_{w b}=x_{w b}+x_{a s}$. Since the parity of degrees at all vertices remain the same except at vertex $\widehat{s}=b$ and the costs of solutions $x$ and $y$ are the same, the optimal $\widehat{s}-\widehat{t}$ traveling salesman walks in $G$ and the optimal $s-t$ traveling salesman walks in $G^{\prime}$ have the same cost. Now, since $G=G^{\prime} \backslash\{s\}$ is walk-perfect, there exists some constraint that is tight for all optimal $\widehat{s}-\widehat{t}$

Fig. $6 u$ is duplicated to obtain $s$ and $t$
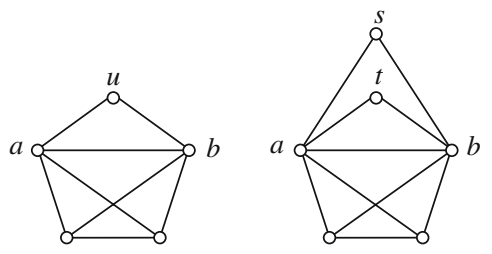
traveling salesman walks in $G$. If this is a non-negativity constraint $y_{e} \geq 0$, then constraint $x_{e} \geq 0$ is also tight for all optimal $s-t$ traveling salesman walks $x$ in $G^{\prime}$. Otherwise, it is a cut constraint $C$ and we can assume without loss of generality that $b \in C$. Then constraint $C^{\prime}=C \cup\{s\}$ is tight for every $x \in \mathcal{P}$.

Case $2.2 c_{a s}, c_{s b}, c_{a t}, c_{t b}>0$.

If $c_{a s}=c_{a t}$ (and therefore $c_{s b}=c_{t b}$ ), then let $F$ be the graph with vertices $V(F)=G^{\prime} \backslash\{s, t\} \cup\{u\}$ and edges $E(F)=E\left(G^{\prime}\right) \cup\{(a, u),(u, b)\}$. Let $y_{a u}=x_{a s}^{*}+x_{a t}^{*}$ and $y_{u b}=x_{s b}^{*}+x_{t b}^{*}$ and $y_{e}=x_{e}$ for all other edges $e$. Then $y$ is a traveling salesman tour on $F$ with cost at most the cost of $x^{*}$ in $G^{\prime}$. Also, any optimal traveling salesman tour on $F$ can be converted to an $s-t$ traveling salesman walk $x$ in $F$ of smaller cost by letting $x_{a s}=y_{a u}, x_{a t}=0, x_{s b}=x_{t b}=y_{u b} / 2$ if $y_{a u}, y_{u b}$ are both even (and therefore equal to 2, by optimality of $y$ ) and $x_{a s}=y_{a u}, x_{a t}=x_{s b}=0, x_{t b}=y_{u b}$ if $y_{a u}, y_{u b}$ are both odd. This shows minimum $s-t$ traveling salesman walks in $G^{\prime}$ and minimum traveling salesman tours in $F$ have the same cost and since $F \simeq G$ is walk-perfect, there is a constraint that is tight for all optimal traveling salesman tours of $F$. If this is a non-negativity constraint $y_{e} \geq 0$, then $x_{e} \geq 0$ is also tight for all $x \in \mathcal{P}$. Otherwise the tight constraint is a cut constraint $C$ and we can assume without loss of generality that $u \in C$. Then $C^{\prime}=C \backslash\{u\} \cup\{s, t\}$ is a tight constraint for all $x \in \mathcal{P}$.

Therefore, $c_{a s} \neq c_{a t}$ and $c_{s b} \neq c_{t b}$. If the inequality $x(\delta(s)) \geq f(s)=1$ is not tight for all $x \in \mathcal{P}$, let $x^{*}$ be an optimal solution with $x^{*}(\delta(s))>1$. Since $\operatorname{deg}(s)$ is odd and $x_{a s}^{*}, x_{s b}^{*}<3$ (by optimality of $x^{*}$ ), we can assume $x_{a s}^{*}=2, x_{s b}^{*}=1$. Then $c_{a s}<c_{a t}$ and $c_{s b}>c_{t b}$ (otherwise, decreasing $x_{a s}^{*}$ by 2 and increasing $x_{a t}^{*}$ by 2 gives a solution of strictly smaller cost). If $\operatorname{deg}(t)=3$, we have the following cases.

Case 2.2.i $x_{a t}^{*} \geq 1, x_{t b}^{*} \geq 1$. In this case, decreasing $x_{a s}^{*}$ by 2 gives an $s-t$ traveling salesman walk of strictly smaller cost, a contradiction.

Case 2.2.ii One of $x_{a t}^{*}, x_{t b}^{*}$ is zero and the other is at least 3 . Then subtracting 2 from the edge of value at least 3 gives an $s-t$ traveling salesman walk of strictly smaller cost, again a contradiction.

Since $\operatorname{deg}(t)$ is odd, it must be the case that $x_{a s}^{*}=2, x_{s b}^{*}=1$ and $\operatorname{deg}(t)=1$. Now, consider the support graph $H=\left\{e \in E\left(V\left(G^{\prime}\right) \backslash\{s, t\}\right): x_{e}^{*}>0\right\}$ and let $x_{H}^{*}$ denote the restriction of $x^{*}$ to this graph. The remaining cases are the following.

Case 2.2.iii $x_{a t}^{*}=1, x_{t b}^{*}=0$. In this case, $x_{H}^{*}$ contains an Eulerian walk from $a$ to $b$ in $H$ since $x_{H}^{*}(w)$ is even for all $w \in H \backslash\{a, b\}$ and odd for $w=a$ or $b$. Therefore, $H$ is connected and a traveling salesman walk of strictly smaller cost can be obtained from $x^{*}$ by decreasing $x_{a s}^{*}$ by 2 .

Case 2.2.iv $x_{a t}^{*}=0, x_{t b}^{*}=1$. If $H$ is connected, the same argument in Case 2.2.iii gives a traveling salesman walk of strictly smaller cost, so we can 
assume $H$ is not connected. Let $C$ be the component of $H$ containing $a$ (note that $b \notin C$ ) and let $C^{\prime}=C \cup\{s\}$. For any edge $e=(i, j) \in E(G)$ with $i \in C, j \notin C$, let $q_{i j}^{s}\left(q_{i j}^{t}\right)$ denote the shortest path in $x^{*}$ from $i$ to $a$ together with edges $(a, s),(s, b)$ (edges $(a, t),(t, b))$ and the shortest path in $x^{*}$ from $b$ to $j$. The cost of edge $e=(i, j)$ must be at least the cost of path $q_{i j}^{s}$ (which is equal to the cost of path $q_{i j}^{t}$ ); otherwise, by replacing path $q_{i j}^{s}$ by edge $(i, j)$, we do not disconnect any vertices of the graph (since $G_{j}$ is a subset of the graph in Fig. 6) while preserving the degree parity at every vertex, which yields an $s-t$ traveling salesman walk of strictly smaller cost.

We claim $x\left(\delta\left(C^{\prime}\right)\right)=1$ for every $x \in \mathcal{P}$. Otherwise, if $x^{*}\left(\delta\left(C^{\prime}\right)\right) \geq 2$ for some $x^{*} \in \mathcal{P}$, then $s \in C^{\prime}, t \notin C^{\prime}$ implies $x^{*}\left(\delta\left(C^{\prime}\right)\right) \geq 3$. One of $x_{s b}^{*}, x_{a t}^{*}$ must be zero, say $x_{a t}^{*}=0$ (otherwise, if $x_{s b}^{*}, x_{a t}^{*} \geq 1$, then decreasing both of these by 1 and increasing $x_{a s}^{*}, x_{t b}^{*}$ by 1 gives an $s-t$ traveling salesman walk of strictly smaller cost). Now, consider edges $\left(k_{1}, l_{1}\right),\left(k_{2}, l_{2}\right),\left(k_{3}, l_{3}\right)$ (possibly including multiple copies of the same edge) crossing $C^{\prime}$ in path $x^{*}$. By rerouting $x_{k_{1} l_{1}}^{*}, x_{k_{2} l_{2}}^{*}$ and $x_{k_{3} l_{3}}^{*}$ along the paths $q_{k_{1} l_{1}}^{s}, q_{k_{2} l_{2}}^{s}$ and $q_{k_{3} l_{3}}^{s}$ (or keeping $x_{k_{i}, l_{i}}^{*}$ if $\left(k_{i}, l_{i}\right)=(s, b)$ ), we obtain an $s-t$ traveling salesman walk $y$ of smaller or equal cost with either $y_{a s} \geq 3$ or $y_{s b} \geq 3$ (if $x_{s b}^{*}=0$, then reroute along the paths $q_{k_{1} l_{1}}^{t}, q_{k_{2} l_{2}}^{t}$ and $\left.q_{k_{3} l_{3}}^{t}\right)$. Now, by decreasing this value by 2 , we obtain an $s-t$ traveling salesman walk of strictly smaller cost, a contradiction. Therefore, $x\left(\delta\left(C^{\prime}\right)\right)=1$ for every $x \in \mathcal{P}$.

We now show that walk perfection for any graph can be reduced to walkperfection of its blocks.

Lemma 4.6 $s-t$ walk-perfection is preserved under operation $\Phi_{1}$.

Proof Suppose vertices $v_{1}$ and $v_{2}$ in connected graphs $G_{1}$ and $G_{2}$ are identified to obtain graph $G$ and let $s, t \in V(G)$. Consider the labeled minor $H_{1}$ obtained by contracting $G_{2}$ to a single vertex in $G$. The result is graph $G_{1}$ where vertex $v_{1}$ has label $s$ if $s \in V\left(G_{2}\right)$, label $t$ if $t \in V\left(G_{2}\right)$, labels $s$ and $t$ if $s, t \in V\left(G_{2}\right)$ and is unlabeled if $s, t \in V\left(G_{1}\right) \backslash\left\{v_{1}\right\}$. Similarly, consider labeled minor $H_{2}$ obtained by contracting $G_{1}$. Since $s-t$ walk-perfection is preserved under connected labeled minors, if $G$ is $s-t$ walk-perfect, then so are $H_{1}$ and $H_{2}$.

Conversely, suppose $H_{1}$ and $H_{2}$ are $s-t$ walk-perfect, let $X(G, s, t)$ denote the set of optimal $s-t$ traveling salesman walks in $G$, and let $x \in X(G, s, t)$. Then optimality and degree parity constraints imply that $x$ is the union of two optimal $s-t$ traveling salesman walks in labeled minors $H_{1}$ and $H_{2}$. For any non-zero cost function $c$, the restriction of $c$ to one of $H_{1}$ or $H_{2}$ must be non-zero; without loss of generality, assume $c$ restricted to $H_{1}$ is non-zero. By Theorem 4.3, there is a constraint $C$ in $P\left(H_{1}\right)$ which is tight for all optimal traveling salesman tours in $H_{1}$. If constraint $C$ is a non-negativity constraint $x_{e} \geq 0$, then this edge constraint is tight for all $x \in X_{T S P}\left(H_{1}\right)$. Otherwise, we can assume constraint $C$ is a cut constraint with $v_{1} \notin C$; in this case, $C$ is a tight constraint for all $x \in X_{T S P}(G)$. 
Now, any 1-connected graph is $C_{6}$ minor free if and only if can be built by repeated applications of $\Phi_{1}$ from blocks which are $C_{6}$ minor free. Therefore, our main theorem follows from Lemmas 4.2, 4.4, 4.5, and 4.6.

Theorem 2.3 A connected graph $G$ is walk-perfect if and only if $G$ has no $C_{6}$ minor.

\section{Connection with TSP-perfection}

In this section, we establish a connection between walk-perfection and TSP-perfection. Using this connection and the characterization of TSP-perfect graphs, we give a second proof of the characterization of walk-perfect graphs.

If graph $G$ is walk-perfect, then it is also TSP-perfect, since by choosing $s=t$, $s-t$ walk-perfection corresponds to TSP-perfection. We would like a condition in the reverse direction, i.e., a sufficient condition for walk-perfection based on TSP-perfection. For graph $G=(V, E)$ and vertices $s, t \in V$, let $G_{s, t}(3)$ denote the graph obtained from $G$ by adding a 3-edge ear between $s$ and $t$ (see Fig. 7).

$$
\begin{gathered}
V\left(G_{s, t}(3)\right)=V(G) \cup\{u, v\}(u, v \notin V(G)) \\
E\left(G_{s, t}(3)\right)=E \cup\{(s, u),(u, v),(v, t)\} .
\end{gathered}
$$

Consider the fractional TSP polyhedron

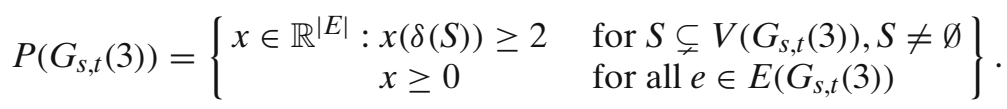

The following lemma relates the extreme points of the fractional traveling salesman walk polyhedron $P(G, s, t)$ with the extreme points of the fractional traveling salesman polyhedron $P\left(G_{s, t}(3)\right)$.

Lemma 5.1 If $x \in \mathbb{R}^{|E|}$ is an extreme point of $P(G, s, t)$, then $x^{\prime}=(x, 1,1,1) \in$ $\mathbb{R}^{\left|E\left(G_{s, t}(3)\right)\right|}$ is an extreme point of $P\left(G_{s, t}(3)\right)$, where the three additional variables correspond to edges $(s, u),(u, v)$, and $(v, t)$.

Proof Let $x$ be an extreme point of $P(G, s, t)$. Then it is tight for $m=|E|$ of the constraints in $P(G, s, t)$. We will show that the point $x^{\prime}=(x, 1,1,1)$ is the unique

Fig. 7 Graph $G_{s, t}(3)$

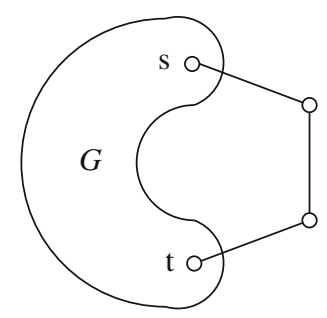


solution to a set of $m+3$ inequalities involving edges $E\left(G_{s, t}(3)\right)$ and therefore is an extreme point $P\left(G_{s, t}(3)\right)$.

First, we show the $m$ tight constraints for $x$ in $P(G, s, t)$ generate $m$ tight constraints for $x^{\prime}$ in $P\left(G_{s, t}(3)\right)$. Each tight constraint $x(\delta(S))=f(S)$ in $P(G, s, t)$ gives rise to a tight constraint $x^{\prime}\left(\delta\left(S^{\prime}\right)\right)=2$ in $P\left(G_{s, t}(3)\right)$ with

$$
S^{\prime}= \begin{cases}S \cup\{u, v\} & \text { if } s, t \in S \\ S^{\prime}=S \cup\{u\} & \text { if } s \in S, t \in \bar{S} \\ S^{\prime}=S \cup\{v\} & \text { if } s \in \bar{S}, t \in S \\ S^{\prime}=S & \text { if } s, t \in \bar{S} .\end{cases}
$$

This gives $m$ tight constraints for $x^{\prime}$ in $P\left(G_{s, t}(3)\right)$. Consider these constraints together with the following three inequalities:

$$
\begin{array}{r}
x(\delta(V))=x_{s u}+x_{v t} \geq 2 \\
x(\delta(V \cup\{u\}))=x_{u v}+x_{v t} \geq 2 \\
x(\delta(V \cup\{v\}))=x_{s u}+x_{u v} \geq 2 .
\end{array}
$$

The unique solution on edges $(s, u),(u, v),(v, t)$ satisfying the last three inequalities at equality is $x_{s u}=x_{u v}=x_{v t}=1$. Furthermore, since $x$ is the unique solution to the $m$ tight constraints in $P(G, s, t)$, it follows that $x^{\prime}=(x, 1,1,1)$ is the unique solution to the $m+3$ tight constraints in $P\left(G_{s, t}(3)\right)$ and therefore, $x^{\prime}$ is a extreme point of $P\left(G_{s, t}(3)\right)$.

Lemma 5.2 If $G_{s, t}(3)$ is TSP-perfect, then $G$ is $s-t$ walk-perfect. If $G_{s, t}(3)$ is TSP-perfect for every choice of $s$ and $t$, then $G$ is walk-perfect.

Proof By Lemma 5.1, if $x$ is an extreme point of $P(G, s, t)$, then $(x, 1,1,1)$ is an extreme point of $P\left(G_{s, t}(3)\right)$. Since $G_{s, t}(3)$ is TSP-perfect, the extreme point $(x, 1,1,1)$ is a tour of $G_{s, t}(3)$, which corresponds to an $s-t$ traveling salesman walk in $G$ together with the three edges $(s, u),(u, v)$, and $(v, t)$. Thus, the extreme point $x$ corresponds to an $s-t$ traveling salesman walk, implying $G$ is $s-t$ walkperfect. If this holds for every choice of $s$ and $t, G$ is walk-perfect.

Claim 5.3 For any $i \in\{1,2,3\}$ and any edge $e \in M_{i}, M_{i} \backslash\{e\}$ contains $C_{6}$ as a minor.

Proof This follows by inspection of Fig. 1.

Theorem 5.4 If $G$ is $C_{6}$ minor free, then $G_{s, t}(3)$ is $\left[M_{1}, M_{2}, M_{3}\right]$ minor free for any choice of $s$ and $t$.

Proof The theorem is clearly true if $s=t$, so we can assume $s \neq t$. Suppose $G_{s, t}(3)$ contains $M_{i}(i=1,2$, or 3$)$ as a minor and label the edges of $G_{s, t}(3)$ according to whether they are contracted, deleted, or unchanged in the sequence of minor operations to obtain $M_{i}$. Consider the 3-path $(s, u),(u, v),(v, t)$. None 
of these edges can be marked for deletion, since this would imply $G$ contains an $M_{i}$ minor, and therefore a $C_{6}$ minor. If any of these edges is unchanged, then after performing the minor operations to obtain $M_{i}$, deleting this edge would leave a $C_{6}$ minor which must have been contained in $G$, a contradiction. Therefore, all 3 edges $(s, u),(u, v),(v, t)$ must be marked for contraction.

Note that since edge contractions and edge deletions can be performed in any order, we can perform the contraction of edges $(s, u),(u, v),(v, t)$ as the final three steps in the sequence of minor operations. Consider the graph at this stage, with only the three edge contractions remaining and let $G^{\prime}$ denote the subgraph of $G$ with all minor operations on $E(G)$ carried out. At this stage, if edges $(s, u),(u, v),(v, t)$ are contracted in graph $G^{\prime} \cup\{(s, u),(u, v),(v, t)\}$, the result is graph $M_{i}$. Note that the contraction cannot result in any multi-edges. Now, since all vertices in $M_{i}$ have degree at most 3 and no multi-edges arise from the contraction of $(s, u),(u, v),(v, t)$, one of $s$ or $t$ (say $t)$ satisfies $\operatorname{deg}_{G^{\prime}}(t) \leq 1$ in $G^{\prime}$. Let $e$ be the edge adjacent to $t$ in $G^{\prime}$ if $\operatorname{deg}_{G^{\prime}}(t)=1$, and let $e$ be an arbitrary edge in $G^{\prime}$ if $\operatorname{deg}_{G^{\prime}}(t)=0$. Then vertex $t$ has degree 0 in $G^{\prime} \backslash\{e\}$ and degree 1 in graph $\left(G^{\prime} \backslash\{e\}\right) \cup\{(s, u),(u, v),(v, t)\}$ and therefore, any $C_{6}$ minor in the graph $\left(G^{\prime} \backslash\{e\}\right) \cup\{(s, u),(u, v),(v, t)\}$ cannot contain vertices $t, u$, or $v$. By Lemma 5.3, deleting edge $e$ from $G^{\prime}$ results in a graph with a $C_{6}$ minor and since this $C_{6}$ minor does not contain any of $t, u$, or $v$, it is also a minor of graph $G$, a contradiction.

Note that since $K_{5}$ is $C_{6}$ minor free, this provides a second proof for the walk-perfection of $K_{5}$, which can be shown by computational methods (see Sect. 4).

Corollary $5.5 K_{5}$ is walk-perfect.

\section{Asymmetric traveling salesman path problem}

In this section, we consider the traveling salesman path problem from the perspective of approximation algorithms. For the graphical traveling salesman problem on graphs with symmetric edge costs satisfying the triangle inequality, Christofides [3] gave a 3/2-approximation algorithm; despite many attempts to find a better approximation guarantee, improving this factor has remained an open problem for almost thirty years. For the more general traveling salesman walk problem, Hoogeveen [12] studies approximation algorithms for walks on metric instances with symmetric edge costs. For fixed $s$ and $t$, he gives a 5/3approximation for the minimum cost $s-t$ traveling salesman walk and for fixed $s$ (and varying endpoint), he gives a 3/2-approximation for the minimum cost traveling salesman walk starting at $s$. An alternate proof for the 5/3-approximation algorithm for two fixed endpoints is due to Vempala [19] and approximations for other variants of this problem can be found in $[1,11]$.

In this section, we give an approximation algorithm for the asymmetric traveling salesman path problem (ATSPP). In this problem, we have fixed vertices $s$ and $t$ in a graph $G=(V, A)$ with directed arcs and possibly asymmetric arc 
costs. The objective is to find a minimum cost directed Hamiltonian path from $s$ to $t$. For the case $s=t$, Frieze et al. gave a $\log _{2} n$-approximation algorithm for the asymmetric traveling salesman tour problem, which was subsequently improved by Bläser [2] to $0.999 \log _{2} n$ and by Kaplan et al. [13] to $0.842 \log _{2} n$.

The ATSW problem is to find a minimum cost directed walk from $s$ to $t$ that visits all vertices at least once. This problem is equivalent to finding a minimum cost directed Hamiltonian path from $s$ to $t$ in the metric completion of graph $G$. Therefore, we focus our attention on complete graphs satisfying the triangle inequality and assume we are given such an instance in our approximation algorithm. Our results are stated for the ATSPP, but apply to the ATSW problem by replacing each arc $(i, j)$ in the solution with a shortest directed path in the graph from $i$ to $j$.

In the following example, we show that there are graphs for which the cost of the optimal asymmetric traveling salesman tour can be arbitrarily higher than that of the optimal asymmetric traveling salesman path. Thus, an $\alpha$-approximation algorithm for the asymmetric traveling salesman tour problem does not immediately yield an $\alpha$-approximation for the ATSPP.

Example 6.1 Figure 8 shows an instance for which the value of the minimum cost tour is arbitrarily higher than the value of the minimum cost $s-t$ traveling salesman path. For this graph, arc $(t, s)$ has arbitrarily high cost $c_{t s}=\alpha$, solid directed arcs have cost 1 and all remaining arcs have costs determined by metric completion. The minimum cost $s-t$ path has value 10 and the minimum cost tour has value $\alpha+10$.

However, using a technique based on recursively building the asymmetric $s-t$ traveling salesman path, we prove that there is an $O(\sqrt{n})$-approximation algorithm for the ATSPP.

\subsection{Path/cycle covers}

An s-t-path/cycle cover in a directed graph $G$ is a directed path from $s$ to $t$ together with a collection of directed cycles such that every vertex in $V$ is contained in exactly one of these subgraphs. In particular, this implies the path and cycles must be disjoint and cover all vertices $V(G)$. Note that the value of the minimum $s-t$-path/cycle cover on $G$ is a lower bound on the minimum cost asymmetric traveling salesman path in $G$. We first show that we can find a minimum $s-t$ path/cycle cover for $G$ efficiently via a reduction to the minimum cost perfect matching problem.

Fig. 8 Example showing an $\alpha$-approximation algorithm for the ATSP does not give an $\alpha$-approximation for the ATSPP

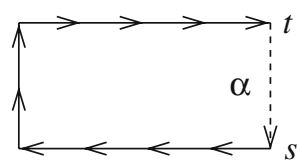


Construct bipartite graph $G^{\prime}$ by including two copies of each vertex $v \in$ $V \backslash\{s, t\}$; call these copies $v$ and $v^{\prime}$. For each pair $i, j \in V \backslash\{s, t\}$, assign cost $c_{i j}$ to $\operatorname{arc}\left(i, j^{\prime}\right)$. Now, include vertices $s$ and $t^{\prime}$ and for all $i \in V \backslash\{s, t\}$, assign cost $c_{s i}$ to $\operatorname{arc}\left(s, i^{\prime}\right)$ and $c_{i t}$ to $\operatorname{arc}\left(i, t^{\prime}\right)$.

Lemma 6.2 The cost of a minimum cost perfect matching in $G^{\prime}$ is equal to the cost of a minimum s-t-path/cycle cover in $G$.

Proof Let $d^{-}(v)$ and $d^{+}(v)$ denote the indegree and outdegree of vertex $v$ respectively. An $s-t$-path/cycle cover is a subgraph of $G$ in which vertices $s$ and $t$ satisfy $d^{+}(s)=d^{-}(t)=1$ and $d^{-}(s)=d^{+}(t)=0$, and every vertex $v \in V \backslash\{s, t\}$ satisfies $d^{-}(v)=d^{+}(v)=1$. We first show that every $s-t$-path/cycle cover of $G$ corresponds to a matching in $G^{\prime}$ with the same cost. For every directed arc $(i, j)$ in the $s-t$-path/cycle cover, include $\operatorname{arc}\left(i, j^{\prime}\right)$ in the matching. Since every vertex in $i \in V \backslash\{s, t\}$ has in-degree 1 and out-degree 1, both $i$ and $i^{\prime}$ are matched in $G^{\prime}$ and since $s$ has out-degree 1 and $t$ has in-degree $1, s$ and $t$ are also matched. Thus, there is a minimum cost perfect matching with the same cost as the $s-t$ path/cycle cover. Conversely, a minimum cost perfect matching in $G^{\prime}$ yields a $s-t$-path/cycle cover in $G$ with the same cost; for every arc $\left(i, j^{\prime}\right)$ in the matching, include arc $(i, j)$ in the path/cycle cover.

\section{$6.2 O(\sqrt{n})$-approximation}

The first step of the algorithm is to find a minimum cost $s-t$-path/cycle cover. If this subgraph contains at least $\sqrt{n}$ cycles, then let $V^{\prime} \subset V$ be the set of vertices in the path together with one vertex from each cycle and let $G^{\prime}$ be the graph induced by the vertices in $V^{\prime}$ (note that $\left|V^{\prime}\right| \leq n-\sqrt{n}$ ). We then recurse on the graph $G^{\prime}$. Such a recursion can occur at most $\sqrt{n}$ times. When we reach a stage in which the path/cycle cover returns fewer than $\sqrt{n}$ cycles, then we attach each cycle to the path resulting in a single $s-t$ path.

This attachment operation proceeds as follows. For each cycle, pick an arbitrary vertex $v$ in the cycle. The current $s-t$ path contains an arc $(a, b)$ such that in an optimal $s-t$ traveling salesman path $\vec{p}$, vertex $v$ falls after $a$ and before $b$. To see why this is true, label all vertices in the current $s-t$ path that appear after $v$ in $\vec{p}$ by 1 and label all vertices that appear before $v$ in $\vec{p}$ by 0 . Then $s$ has label 0 and $t$ has label 1 and therefore, there is some arc $(a, b)$ such that $a$ has label 0 and $b$ has label 1 . Although we do not know which arc will satisfy the desired property, we can test all consecutive vertices along the $s-t$ path and choose $a$ and $b$ to minimize the length of the sum of the two arcs $(a, v)$ and $(v, b)$. Then by connecting vertex $v$ to the $s-t$ path by adding these two arcs, the cost incurred is at most OPT (see Fig. 9).

Since there are at most $k \leq \sqrt{n}$ cycles, the total cost of adding all these arcs is at most $\sqrt{n} \cdot O P T$. In the final step, we have an $s-t$-path on a subset of the vertices and we expand each vertex that represented a cycle at some stage of the algorithm by replacing the vertex with a complete traversal of that cycle. If a vertex $v$ is visited multiple times in the result, then let $(i, v)$ and $(v, j)$ be two 
Fig. 9 Attaching the cycles to the path

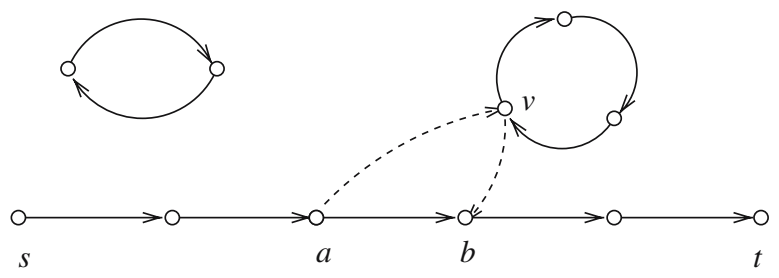

arcs in the solution. Since the graph is assumed to be a complete directed graph satisfying the triangle inequality, we can shortcut the solution by including arc $(i, j)$ and deleting arcs $(i, v)$ and $(v, j)$. Repeating this procedure until every vertex is visited exactly once results in a directed $s-t$ traveling salesman path.

\section{$\operatorname{ATSPP}-\operatorname{Approx}(G)$}

1. Find a minimum cost $s-t$ path/cycle cover $C$ for $G$.

(i) If $C$ has less than $\sqrt{n}$ cycles, then attach the cycles to the $s-t$ path and let $S$ be the resulting path.

(ii) Else if $C$ has more than $\sqrt{n}$ cycles, then let $V^{\prime}$ be the set of vertices in the $s-t$ path plus one representative vertex from each cycle. Run $\operatorname{ATSPP}-\operatorname{ApPROx}\left(G^{\prime}\right)$ for $G^{\prime}=\left(V^{\prime}, A\left(V^{\prime}\right)\right)$.

2. For each vertex that represents a cycle in $S$, expand the cycle while traversing the path, shortcutting arcs through vertices which are visited multiple times.

Acknowledgements The authors would like to thank Santosh Vempala for suggesting problems on traveling salesman paths, Kevin Cheung, Michel Goemans, Kunal Talwar Santosh Vempala for many helpful discussions, and the anonymous referees for their helpful comments.

\section{References}

1. Anily, S., Bramel, J., Hertz, A.: A 5/3-approximation algorithm for the cluster traveling salesman tour and path problems. Oper. Res. 24, 29-35 (1999)

2. Bläser, M.: A new approximation algorithm for the asymmetric TSP with triangle inequality. In: Proceedings of the ACM-SIAM symposium on discrete algorithms, pp. 638-645, 2002

3. Christofides, N.: Worst-case analysis of a new heuristic for the traveling salesman problem. Technical report, GSIA, Carnegie Mellon University, 1976

4. Cornu'ejols, G., Fonlupt, J., Naddef, D.: The traveling salesman problem on a graph and some related integer polyhedra. Math. Program. 33, 1-27 (1985)

5. Fleischmann, B.: A cutting plane procedure for the travelling salesman problem on road networks. Eur. J. Oper. Res. 21 (1985)

6. Fonlupt, J., Naddef, D.: The traveling salesman problem in graphs with some excluded minors. Math. Program. 53, 147-172 (1992)

7. Frieze, A., Galbiati, G., Maffioli, F.: On the worst-case performance of some algorithms for the asymmetric traveling salesman problem. Networks 12, 23-39 (1982)

8. Gawrilow, E., Joswig, M.: Polymake: a framework for analyzing convex polytopes, pp. 43-74, 2000

9. Goemans, M. X.: The Steiner tree polytope and related polyhedra. Math. Program. 63,157-182 (1994) 
10. Gutin, G., Punnen, A. (eds.) The traveling salesman problem and its variations. Kluwer, Dordrecht (2002)

11. Guttmann-Beck, N., Hassin, R., Khuller, S., Raghavachari, B.: Approximation algorithms with bounded performance guarantees for the clustered traveling salesman problem. Algorithmica 28, 422-437 (2000)

12. Hoogeveen, J.A.: Analysis of Christofides' heuristic: some paths are more difficult than cycles. Oper. Res. Lett. 10, 291-295 (1991)

13. Kaplan, H., Lewenstein, M., Shafir, N., Sviridenko, M.: Approximation algorithms for asymmetric TSP by decomposing directed regular multigraphs. In: Proceedings of the IEEE Foundations on Computer Science, pp. 56-67, 2003

14. Lam, F.: Traveling salesman path problems, Ph.D thesis, Massachusetts Institute of Technology, 2005

15. Lawler, E.L., Lenstra, J.K., Rinnooy Kan, A.H.G, Shmoys, D.B.: The traveling salesman problem, a guided tour of combinatorial optimization. Wiley, New York (1985)

16. Naddef, D., Rinaldi, G.: The symmetric traveling salesman polytope and its graphical relaxation: composition of valid inequalities. Math. Program. 51, 359-400 (1991)

17. Naddef, D., Rinaldi, G.: The graphical relaxation: a new framework for the symmetric traveling salesman polytope. Math. Program. 58, 53-88 (1993)

18. Schrijver, A.: Combinatorial optimization, polyhedra and efficiency, vols. A,B,C. Springer, Berlin Heidelberg New York (2003)

19. Vempala, S.: Personal communication 